\title{
Joint Supply, Demand, and Energy Storage Management Towards Microgrid Cost Minimization
}

\author{
Sun Sun*, Min Dong ${ }^{\dagger}$, and Ben Liang* \\ ${ }^{*}$ Dept. of Electrical and Computer Engineering, University of Toronto, Canada \\ ${ }^{\dagger}$ Dept. of Electrical, Computer and Software Engineering, University of Ontario Institute of Technology, Canada \\ Email: \{ssun, liang\}@ comm.utoronto.ca, min.dong@uoit.ca
}

\begin{abstract}
The problem of real-time power balancing in a gridconnected microgrid is studied. We consider a microgrid powered by a conventional generator (CG) and multiple renewable generators (RGs) each co-located with one distributed storage (DS) unit. An aggregator operates the microgrid and aims to minimize the long-term system cost, including all RGs' cost, the CG's cost, and the cost for exploiting external energy markets. We jointly manage the supply side, demand side, and DS units, taking into account the randomness of the system, and incorporating the ramping constraint of the CG. A real-time algorithm is proposed, which does not require any statistics of the system. We analytically characterize the gap between the system cost under our algorithm and the minimum cost, demonstrating that our algorithm is asymptotically optimal as the DS energy capacity increases and the CG ramping constraint loosens. In simulation, we compare the proposed algorithm with a greedy algorithm as well as a lower bound on the optimum. Simulation shows that our algorithm outperforms the greedy one and its performance can be close to the optimum even with small DS units.
\end{abstract}

\section{INTRODUCTION}

Microgrid is seen to be the basic structure in the future power grid [1]. With the growing environmental concerns and the need to reduce greenhouse gas emissions, more and more renewable generation, such as wind and solar, is expected to be integrated into the microgrid. However, as renewable generation is intermittent and non-dispatchable, straightforward integration of a large amount of renewable generation would create noticeable variations in power supply. On the other hand, continuously balancing supply and demand is of paramount importance for the reliability of a power system [2]. Therefore, how to efficiently integrate renewable generation while ensuring the system reliability is crucial for microgrids.

A microgrid is typically characterized by distributed generation (DG), distributed storage (DS), and loads. Besides renewable generators (RGs), DG can encompass some conventional generators (CGs) such as nuclear, coal-fired, or gas-fired generators. For DS, its charging and discharging capability can be exploited to regulate the energy supply. Hence, colocation of DS and a RG is often suggested. Moreover, loads generally contain base loads and flexible loads, and the latter can be controlled through either curtailment or time shift. For system reliability, the microgrid has to maintain power balance continuously. This generally involves three types of management: supply side management, demand side management, and storage management, which require the coordination of all components in the system. Designing an efficient algorithm for real-time power balancing in microgrids is challenging. Concretely, since the system states such as the renewable generation, loads, and electricity prices are random, accurate modeling and statistical information may be required, which are difficult to obtain in reality. Second, due to practical operation limitations such as the restriction of the energy capacity of DS units and the ramping constraint of CGs, the control decisions are coupled over time, which complicates the real-time decision making.

Recently, there are many works on energy management in microgrids, emphasizing various aspects of the system. For example, [3] and [4] consider supply side management by assuming all loads are uncontrollable, [5] studies demand side management, while [6] and [7] propose to employ DS units to clear power imbalance in real time. [8] and [9] put these three types of management together. However, in [8], although the uncertainty of the renewable generation is characterized by a polyhedral set, the uncertainty of the loads and electricity prices is ignored. Moreover, the algorithm is designed for offline use such as in day-ahead scheduling, and therefore cannot be implemented in real time. In [9], a real-time algorithm is proposed to minimize the cost of the CG only. Nevertheless, the ramping constraint of the CG is not taken into account.

In this paper, we investigate the problem of real-time power balancing in a microgrid by incorporating supply side management, demand side management, and storage management. We consider a microgrid powered by a CG and multiple RGs each co-located with one DS unit. An aggregator operates the microgrid and aims to minimize the long-term system cost, including all RGs' cost, the CG's cost, and the cost for selling and purchasing energy from the external energy markets. We take the system randomness into account and also incorporate the ramping constraint of the CG. To solve the underlying optimization problem, a real-time algorithm is proposed.

The main contribution of this paper is two-fold. First, we build a more complete microgrid system and formulate the real-time power balancing problem as a stochastic optimization problem. Second, we offer a real-time algorithm, which does not require any statistics of the system and meanwhile provides a strong performance guarantee. In particular, we characterize the gap between the system cost under our algorithm and the minimum cost. We demonstrate that our algorithm is asymptotically optimal as the energy capacity of the DS units increases and the ramping constraint of the CG loosens. In 


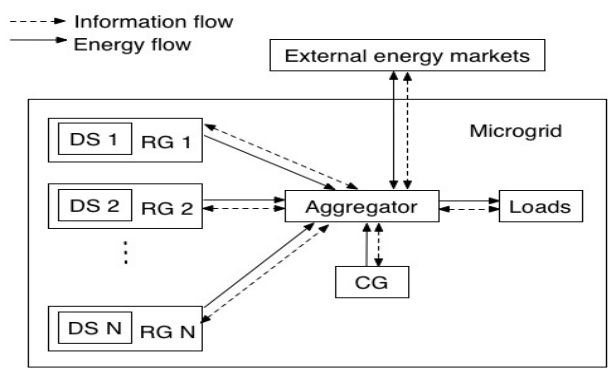

Fig. 1. Schematic representation of a microgrid.

simulation, we compare our algorithm with a greedy algorithm as well as a lower bound on the minimum system cost. Simulation shows that our algorithm outperforms the greedy algorithm and can be close to the minimum cost even with small DS units.

The remainder of this paper is organized as follows. In Section II, we describe the system model of a microgrid and formulate an optimization problem for real-time power balancing. In Section III, we propose a real-time algorithm and in Section IV, we analyze its performance. Simulation results are presented in Section V, and we conclude in Section VI.

\section{System Model and Problem Statement}

\section{A. System Model}

As shown in Fig. 1, we consider a microgrid powered by a CG (e.g., nuclear, coal-fired, or gas-fired generator) and $N$ RGs (e.g., wind or solar) each equipped with one on-site DS unit. The microgrid is connected to the external energy markets and is operated by an aggregator, who is responsible for satisfying the loads by managing energy from various sources. The information flow and the energy flow are also depicted in Fig. 1. Assume that the system operates in discrete time with time slot $t \in\{0,1,2, \ldots\}$. The details of each component in the microgrid are described below.

1) Loads: The loads include base loads and flexible loads. The base loads represent the critical energy demands such as lighting, which must be satisfied once requested. The flexible loads represent the controllable energy requests, such as those from thermostatically controlled loads (TCLs), electric vehicles (EVs), and other smart appliances, which can be partly curtailed if the energy provision cost is high. At time slot $t$, denote the amount of the requested base loads as $l_{b, t} \in\left[l_{b, \min }, l_{b, \max }\right]$ and the amount of the requested flexible loads as $l_{f, t} \in\left[l_{f, \min }, l_{f, \max }\right]$. The amounts $l_{b, t}$ and $l_{f, t}$ are generated by users based on their own needs and are considered random. Let the amount of the total satisfied loads be $l_{m, t}$, which is bounded by $l_{b, t} \leq l_{m, t} \leq l_{b, t}+l_{f, t}$.

The control of the flexible loads needs to meet certain quality-of-service requirement. In this work, we impose an upper bound on the portion of the unsatisfied flexible loads. Formally, we introduce a long-term constraint $\lim \sup _{T \rightarrow \infty} \frac{1}{T} \sum_{t=0}^{T-1} \mathbb{E}\left[\frac{l_{b, t}+l_{f, t}-l_{m, t}}{l_{f, t}}\right] \leq \alpha$ where $\alpha \in[0,1]$ is a pre-designed threshold with a smaller value indicating a tighter quality-of-service requirement.
2) $R G$ and On-Site DS Unit: At the $i$-th RG, denote the amount of the renewable generation during time slot $t$ by $a_{i, t} \in\left[0, a_{i, \max }\right]$, where $a_{i, \max }$ is the maximum generated energy amount. Due to the stochastic nature of the renewable sources, $a_{i, t}$ is random over time. To mitigate such randomness, we assume that each RG is co-located with one onsite DS unit capable of charging and discharging. Denote the charging/discharging energy amount of the $i$-th DS unit during time slot $t$ as $x_{i, t}$, with $x_{i, t}>0(<0)$ indicating charging (discharging). Owing to the storage battery characteristics, $x_{i, t}$ is bounded within $\left[x_{i, \min }, x_{i, \max }\right]$ with $\left|x_{i, \min }\right|$ and $x_{i, \max }$ representing the maximum discharging and charging amount, respectively.

In every time slot, the RG supplies energy to the aggregator. Denote $b_{i, t} \geq 0$ as the amount of the contributed energy by the $i$-th RG during time slot $t$. Since the energy flows of the RG should be balanced, we have $b_{i, t}=a_{i, t}-x_{i, t}, \forall t$. In particular, if $x_{i, t}>0$ (charging), the energy contribution amount $b_{i, t}$ comes from part of the renewable generation; if $x_{i, t}<0$ (discharging), $b_{i, t}$ comes from both the renewable generation and the DS unit.

For the $i$-th DS unit, denote its energy state at the beginning of time slot $t$ by $s_{i, t}$ with the initial energy state $s_{i, 0}=0$. Due to charging and discharging operations, the energy state fluctuates over time and evolves as follows ${ }^{1}$ :

$$
s_{i, t+1}=s_{i, t}+x_{i, t}, \forall t .
$$

It is known that fast charging/discharging causes battery degradation, which shortens battery lifetime [10]. Moreover, faster charging/discharging (i.e., larger $\left|x_{i, t}\right|$ ) generally has a more detrimental effect on battery lifetime. To model this effect on the $i$-th DS unit, we use $D_{i}(\cdot)$ to represent the degradation cost function associated with the charging/discharging amount $x_{i, t}$. We assume $D_{i}(\cdot)$ to be convex, non-increasing on $\left[x_{i, \min }, 0\right]$, and non-decreasing on $\left[0, x_{i, \max }\right]$. To facilitate later analysis, we add a mild condition that $D_{i}(\cdot)$ is continuously differentiable with a bounded first derivative, i.e., $D_{i, \min }^{\prime} \leq D_{i}^{\prime}\left(x_{i, t}\right) \leq D_{i, \max }^{\prime}, \forall x_{i, t} \in\left[x_{i, \min }, x_{i, \max }\right]$.

3) $C G$ : Besides the RGs, the aggregator can coordinate a dispatchable CG. Denote $g_{t} \in\left[0, g_{\max }\right]$ as the output of the CG during time slot $t$, where $g_{\max }$ is the maximum amount of the energy output. Due to practical limitations of the CG, the change of its outputs in two consecutive time slots cannot be arbitrarily large, but is bounded. This is typically reflected by a ramping constraint on the CG outputs [11]. Assume that the ramp-up and the ramp-down constraints are identical. Then, we can express the overall ramping constraint as $\left|g_{t}-g_{t-1}\right| \leq$ $r g_{\max }$ where the coefficient $r \in[0,1]$ indicates the tightness of the ramping requirement. In particular, if $r=0$, the $\mathrm{CG}$ produces a fixed output over time, and if $r=1$, the ramping requirement becomes non-effective.

The generation cost function of the CG is denoted by $C(\cdot)$, which is assumed to be convex and non-decreasing on

\footnotetext{
${ }^{1}$ In this work we use a simplified energy storage model. Our mathematical framework carries over when other modeling factors such as charging/discharging efficiency and storage leakage are considered.
} 
$\left[0, g_{\max }\right]$. Furthermore, we assume $C(\cdot)$ to be continuously differentiable with a bounded first derivative, i.e., $C_{\min }^{\prime} \leq$ $C^{\prime}\left(g_{t}\right) \leq C_{\text {max }}^{\prime}, \forall g_{t} \in\left[0, g_{\text {max }}\right]$.

4) External Energy Markets: In addition to the internal energy transactions, the aggregator can resort to the external energy markets if needed. For example, the aggregator can buy energy from the external energy markets in the case of energy deficit, or sell energy to the markets in the case of energy surplus. At time slot $t$, denote the unit prices of the external energy markets for buying and selling energy as $p_{b, t} \in\left[p_{b, \min }, p_{b, \max }\right]$ and $p_{s, t} \in\left[p_{s, \min }, p_{s, \max }\right]$, respectively. To avoid arbitrage, the buying price is strictly greater than the selling price, i.e., $p_{b, t}>p_{s, t}, \forall t$. Note that the prices $p_{b, t}$ and $p_{s, t}$ are typically random due to unexpected market behaviors. Denote $e_{b, t} \geq 0$ and $e_{s, t} \geq 0$ as the amount of the energy bought from and sold to the external energy markets during time slot $\mathrm{t}$, respectively.

5) System State and Constraint: Based on the previously described system components, we define the system state at time slot $t$ as

$$
\mathbf{q}_{t} \triangleq\left[\mathbf{a}_{t}, l_{b, t}, l_{f, t}, p_{b, t}, p_{s, t}\right]
$$

where $\mathbf{a}_{t} \triangleq\left[a_{1, t}, \cdots, a_{N, t}\right]$. We assume $\mathbf{q}_{t}$ to be i.i.d. over time, but the elements within $\mathbf{q}_{t}$ can be arbitrarily correlated. For system reliability, the energy should be balanced at all times, which yields a system-wide balancing constraint $g_{t}+e_{b, t}+\sum_{i=1}^{N} b_{i, t}=e_{s, t}+l_{m, t}, \forall t$. To the aggregator, the left hand side represents the amount of the total incoming energy, while the right hand side represents the amount of the total outgoing energy.

\section{B. Problem Statement}

The aggregator controls the energy flows in the microgrid. It aims to minimize the long-term time-averaged system cost by jointly managing the supply side, demand side, and storage. In particular, the decision vector at time slot $t$ is defined by

$$
\mathbf{u}_{t} \triangleq\left[\mathbf{b}_{t}, \mathbf{x}_{t}, l_{m, t}, g_{t}, e_{b, t}, e_{s, t}\right]
$$

where $\mathbf{b}_{t} \triangleq\left[b_{1, t}, \cdots, b_{N, t}\right]$ and $\mathbf{x}_{t} \triangleq\left[x_{1, t}, \cdots, x_{N, t}\right]$. The system cost at time slot $t$ includes all RGs' cost, the CG's cost, and the cost for exploiting the external energy markets, which is defined as follows ${ }^{2}$ :

$$
w_{t} \triangleq\left[\sum_{i=1}^{N} D_{i}\left(x_{i, t}\right)\right]+C\left(g_{t}\right)+p_{b, t} e_{b, t}-p_{s, t} e_{s, t} .
$$

Based on the system model described in Section II-A, we formulate the problem as a stochastic optimization problem:

$$
\begin{aligned}
& \text { P1 : } \min _{\left\{\mathbf{u}_{t}\right\}} \quad \limsup _{T \rightarrow \infty} \frac{1}{T} \sum_{t=0}^{T-1} \mathbb{E}\left[w_{t}\right] \\
& \text { s.t. } \quad l_{b, t} \leq l_{m, t} \leq l_{b, t}+l_{f, t}, \quad \forall t,
\end{aligned}
$$

\footnotetext{
${ }^{2}$ For the RGs and the CG, the payment for supplying energy could be settled by additional contracts offered by the aggregator, or be calculated based on the actual provided energy. For both of these cases, the payment is transfered inside the system hence not affecting the system-wide cost.
}

$$
\begin{aligned}
& x_{i, \min } \leq x_{i, t} \leq x_{i, \max }, \quad \forall i, t, \\
& 0 \leq s_{i, t}<\infty, \quad \forall i, t, \\
& b_{i, t} \geq 0, \quad \forall i, t, \\
& b_{i, t}=a_{i, t}-x_{i, t}, \quad \forall i, t, \\
& 0 \leq g_{t} \leq g_{\max }, \quad \forall t, \\
& \left|g_{t}-g_{t-1}\right| \leq r g_{\max }, \quad \forall t, \\
& e_{b, t} \geq 0, \quad e_{s, t} \geq 0, \quad \forall t, \\
& g_{t}+e_{b, t}+\sum_{i=1}^{N} b_{i, t}=e_{s, t}+l_{m, t}, \forall t, \\
& \limsup _{T \rightarrow \infty} \frac{1}{T} \sum_{t=0}^{T-1} \mathbb{E}\left[\frac{l_{b, t}+l_{f, t}-l_{m, t}}{l_{f, t}}\right] \leq \alpha
\end{aligned}
$$

where the expectations in the objective and (11) are taken over the randomness of the system state $\mathbf{q}_{t}$ and the possibly random control decision $\mathbf{u}_{t}$ in each time slot. Constraint (4) requires that the energy state of each DS unit be always bounded. Note that the system cost depends on the capacity of each DS unit. For now we do not specify the capacity of each DS unit, and will discuss it later in Section IV.

We emphasize that, solving $\mathbf{P 1}$ is challenging. Specifically, first, the system state $\mathbf{q}_{t}$ is random. This implies that we may need accurate system modeling and statistical information (such as in dynamic programing), which are difficult to obtain in practice. Second, constraints (4), (8), and (11) couple the decision $\mathbf{u}_{t}$ over time, further complicating the management. In this paper, we aim to propose an algorithm that is easy to implement and does not require any system statistics.

\section{ReAl-Time Algorithm For Microgrid Power BALANCING}

In this section, leveraging Lyapunov optimization techniques [12], we propose a real-time algorithm for solving P1.

\section{A. Problem Relaxation}

Lyapunov optimization can be used to transform timeaveraged constrains such as (11) into queue stability constraints, and to provide simple real-time algorithms for complex dynamic systems. Unfortunately, the energy state constraint (4) and the ramping constraint (8) are not time-averaged constraints, but are hard constraints required in each time slot. These two constraints couple the decision over time and make P1 a more challenging problem that cannot be directly handled by the standard Lyapunov optimization techniques. To overcome this difficulty, we first relax P1 to the following problem.

$$
\begin{aligned}
\text { P2: } \min _{\left\{\mathbf{u}_{t}\right\}} & \limsup _{T \rightarrow \infty} \frac{1}{T} \sum_{t=0}^{T-1} \mathbb{E}\left[w_{t}\right] \\
\text { s.t. } & (2)(3),(5)-(7),(9)-(11), \\
& \lim _{T \rightarrow \infty} \frac{1}{T} \sum_{t=0}^{T-1} \mathbb{E}\left[x_{i, t}\right]=0, \quad \forall i .
\end{aligned}
$$


Compared with P1, in P2, the energy state constraint (4) is replaced with a new time-averaged constraint (12) and the ramping constraint (8) is removed. Using the energy state update in (1) and $s_{i, 0}=0, \forall i$, we can derive that the left hand side of constraint (12) is identical to the limiting value of $\frac{\mathbb{E}\left[s_{i, T}\right]}{T}$, i.e.,

$$
\lim _{T \rightarrow \infty} \frac{1}{T} \sum_{t=0}^{T-1} \mathbb{E}\left[x_{i, t}\right]=\lim _{T \rightarrow \infty} \frac{\mathbb{E}\left[s_{i, T}\right]}{T} .
$$

From (13), if $s_{i, t}$ is always bounded, i.e., constraint (4) holds, then constraint (12) is satisfied. Therefore, $\mathbf{P 2}$ is indeed a relaxed problem of $\mathbf{P} \mathbf{1}$.

We need to point it out that this relaxation transformation is crucial and enables us to apply the standard Lyapunov techniques. However, the solution of $\mathbf{P 2}$ may be infeasible to P1. In the next subsection, based on P2, we propose a realtime algorithm which ensures to produce a feasible solution to $\mathbf{P 1}$.

\section{B. Real-Time Algorithm}

At time slot $t$, define a vector $\boldsymbol{\Theta}_{t} \triangleq\left[s_{1, t}, \cdots, s_{N, t}, J_{t}\right]$, which consists of the energy states of all DS units and a virtual queue backlog $J_{t}$. In particular, $J_{t}$ is associated with the timeaveraged constraint (11) and evolves as follows:

$$
J_{t+1}=\max \left\{J_{t}-\alpha, 0\right\}+\frac{l_{b, t}+l_{f, t}-l_{m, t}}{l_{f, t}}, \forall t .
$$

From (14), the virtual queue $J_{t}$ accumulates the portion of the unsatisfied flexible loads. We initialize it as $J_{0}=0$. Based on constraint (2), we have $J_{t} \geq 0, \forall t$.

Using $\boldsymbol{\Theta}_{t}$, we define the Lyapunov function as

$$
L\left(\boldsymbol{\Theta}_{t}\right) \triangleq \frac{1}{2} J_{t}^{2}+\frac{1}{2} \sum_{i=1}^{N}\left(s_{i, t}-\beta_{i}\right)^{2}
$$

where $\beta_{i}$ is a perturbation parameter associated with the $i$-th DS unit and is defined as

$$
\beta_{i} \triangleq V\left(p_{b, \max }+D_{i, \max }^{\prime}\right)-x_{i, \min }
$$

with $V>0$ being a control parameter. Note that the design of $\beta_{i}$ is important and guarantees the boundedness of $s_{i, t}$. We will discuss more about this related issue on $s_{i, t}$ in Section IV. The idea of the perturbation parameter in Lyapunov optimization is originally introduced in [13]. Nevertheless, [13] studies the scheduling of packet transmissions for energy harvesting networks, which is intrinsically different from our problem. Therefore, the specific design of $\beta_{i}$ in our problem is different from that in [13].

We define the one-slot conditional Lyapunov drift as $\Delta\left(\boldsymbol{\Theta}_{t}\right) \triangleq \mathbb{E}\left[L\left(\boldsymbol{\Theta}_{t+1}\right)-L\left(\boldsymbol{\Theta}_{t}\right) \mid \boldsymbol{\Theta}_{t}\right]$. Moreover, we define the drift-plus-cost function as $\Delta\left(\boldsymbol{\Theta}_{t}\right)+V \mathbb{E}\left[w_{t} \mid \boldsymbol{\Theta}_{t}\right]$, which is a weighted sum of the one-slot conditional Lyapunov drift and the objective function. In the following lemma, we show that the drift-plus-cost function is upper bounded.
Lemma 1: For all possible decisions and all possible values of $\boldsymbol{\Theta}_{t}$, in each time slot $t$, the drift-plus-cost function is upper bounded as follows

$$
\begin{aligned}
\Delta\left(\boldsymbol{\Theta}_{t}\right) & +V \mathbb{E}\left[w_{t} \mid \boldsymbol{\Theta}_{t}\right] \leq B+J_{t} \mathbb{E}\left[\frac{l_{b, t}+l_{f, t}-l_{m, t}}{l_{f, t}}-\alpha \mid \boldsymbol{\Theta}_{t}\right] \\
& +\sum_{i=1}^{N}\left(s_{i, t}-\beta_{i}\right) \mathbb{E}\left[x_{i, t} \mid \boldsymbol{\Theta}_{t}\right]+V \mathbb{E}\left[w_{t} \mid \boldsymbol{\Theta}_{t}\right]
\end{aligned}
$$

where $B$ is a constant and is given by $B \triangleq \frac{1}{2}\left(1+\alpha^{2}\right)+$ $\frac{1}{2} \sum_{i=1}^{N} \max \left\{x_{i, \min }^{2}, x_{i, \max }^{2}\right\}$.

Proof: See Appendix A of our technical report [14].

The proposed real-time algorithm is to minimize the right hand side of inequality (17) in every time slot. The hope is that, through the minimization of the upper bound in every time slot, we can actually minimize the Lyapunov function (hence the queue backlog $J_{t}$ and $\left.\left|s_{i, t}-\beta_{i}\right|\right)$ and the objective function together. Nevertheless, since the ramping constraint (8) has been dropped in the relaxed problem P2, the optimal solution of $g_{t}$ may violate constraint (8). To avoid the infeasibility of $g_{t}$, we move constraint (8) back into the real-time problem. That is, we now solve the following problem at time slot $t$.

$$
\begin{aligned}
\mathbf{P 3}: \min _{\mathbf{u}_{t}} & {\left[\sum_{i=1}^{N} V D_{i}\left(x_{i, t}\right)+\left(s_{i, t}-\beta_{i}\right) x_{i, t}\right]+V C\left(g_{t}\right) } \\
& +V p_{b, t} e_{b, t}-V p_{s, t} e_{s, t}-\frac{J_{t}}{l_{f, t}} l_{m, t} \\
\text { s.t. } & (2)(3),(5)-(10) .
\end{aligned}
$$

Therefore, the solution of $g_{t}$ in $\mathbf{P 3}$ is always feasible to P1.

Based on the description above, we summarize the proposed algorithm as follows. Clearly, no system statistics is required, which makes it easy to implement in reality.

Algorithm 1 Real-time microgrid power balancing.

Initializes $J_{0}=0$. At each time slot, the aggregator executes the following steps sequentially.

1: Observe the system state $\mathbf{q}_{t}$, energy state $s_{i, t}, \forall i$, and queue backlog $J_{t}$.

2: Solve the real-time problem $\mathbf{P 3}$.

3: Update $s_{i, t}, \forall i$, and $J_{t}$ based on (1) and (14), respectively.

\section{Performance Analysis}

In this section, we analyze the performance of the proposed algorithm with respect to the original problem P1.

Denote the decision at time slot $t$ under Algorithm 1 by $\mathbf{u}_{t}^{*} \triangleq\left[\mathbf{b}_{t}^{*}, \mathbf{x}_{t}^{*}, l_{m, t}^{*}, g_{t}^{*}, e_{b, t}^{*}, e_{s, t}^{*}\right]$. In addition, to emphasize the dependence of the system cost with the ramping coefficient $r$ and the control parameter $V$, we denote the system cost under Algorithm 1 by $w^{*}(r, V)$. Denote the minimum system cost of $\mathbf{P 1}$ by $w^{\mathrm{opt}}(r)$, which is independent of $V$.

We now show that the energy state of each DS unit under our algorithm satisfies the boundedness constraint (4) in P1. To this end, we first give the following lemma. It describes a 
sufficient condition under which the $i$-th DS unit will charge or discharge.

Lemma 2:

1) If $s_{i, t}<-x_{i, \min }$, then $x_{i, t}^{*}=\min \left\{a_{i, t}, x_{i, \max }\right\}$.

2) If $s_{i, t}>\beta_{i}-V\left(p_{s, \min }+D_{i, \min }^{\prime}\right)$, then $x_{i, t}^{*}=x_{i, \min }$.

Proof: See Appendix B of our technical report [14].

Lemma 2 conveys that if the energy state $s_{i, t}$ is too low the DS unit will charge, and if $s_{i, t}$ is too high the DS unit will discharge. This lemma provides the key results for demonstrating the boundedness of $s_{i, t}$. Using Lemma 2, we can prove that $s_{i, t}$ is confined within a specific interval.

Lemma 3: For the $i$-th DS unit, the energy state $s_{i, t}$ is always confined within the interval $\left[0, s_{i, \max }\right]$ where

$$
\begin{aligned}
s_{i, \max } \triangleq & V\left(p_{b, \max }-p_{s, \text { min }}+D_{i, \max }^{\prime}-D_{i, \min }^{\prime}\right) \\
& -x_{i, \min }+x_{i, \max } .
\end{aligned}
$$

Proof: See Appendix C of our technical report [14].

Lemma 3 is quite informative and reveals a linear relationship between $s_{i, \max }$ and the control parameter $V$. In particular, by setting $s_{i, \max }$ as the energy capacity of the $i$-th DS unit based on the definition in (18), we can ensure that constraint (4) in P1 holds. On the other hand, if $s_{i, \max }$ is known, the boundedness of $s_{i, t}$ can be guaranteed by appropriately setting the control parameter $V$. The following proposition is obtained straightforwardly from Lemma 3.

Proposition 1: Assume that the energy capacity of the $i$ th DS unit $s_{i, \max }$ is known. Then, by setting $V \in\left(0, V_{\max }\right]$ where

$$
V_{\max } \triangleq \min _{1 \leq i \leq N}\left\{\frac{s_{i, \max }+x_{i, \min }-x_{i, \max }}{p_{b, \max }-p_{s, \min }+D_{i, \max }^{\prime}-D_{i, \min }^{\prime}}\right\},
$$

we have $s_{i, t} \in\left[0, s_{i, \max }\right], \forall t$.

Note that Lemmas 2 and 3, and Proposition 1 are all sample path results that do not depend on the statistics of the system. Next, we characterize the time-averaged system cost of Algorithm 1 with respect to P1.

Theorem 1: Assume that the system state $\mathbf{q}_{t}$ is i.i.d. over time $^{3}$ and the energy capacity of each DS unit is given by $s_{i, \max }$ based on (18). Then, under Algorithm 1,

1) the decisions $\left\{\mathbf{u}_{t}^{*}\right\}$ are feasible to $\mathbf{P 1}$;

2) $w^{*}(r, V)$ is upper bounded as

$w^{*}(r, V) \leq w^{\mathrm{opt}}(r)+B / V+(1-r) g_{\max } \max \left\{p_{b, \max }, C_{\max }^{\prime}\right\}$

where $B$ has been defined below (17); and

3) $w^{\text {opt }}(r)$ is lower bounded as $w^{\text {opt }}(r) \geq w^{*}(1, V)-B / V$.

Proof: See Appendix D of our technical report [14].

Theorem 1.1) indicates that, despite the relaxation to $\mathbf{P 2}$, the feasibility of the solution is maintained under our algorithm.

In Theorem 1.2), we characterize the gap between the system cost under Algorithm 1 and the minimum cost of P1. Different from the standard $O(1 / V)$ gap in typical Lyapunov optimization [12], here, the ramping constraint introduces an

\footnotetext{
${ }^{3}$ Using a multi-slot drift technique, we can show similar conclusions when $\mathbf{q}_{t}$ evolves based on a finite state irreducible and aperiodic Markov chain.
}

extra additive gap that increases linearly with $(1-r)$. The upper bound in Theorem 1.2) reflects that Algorithm 1 is asymptotically optimal as $V$ (or the energy capacity of the DS units) increases and the ramping constraint becomes loose (i.e., $r \rightarrow 1$ ).

In Theorem 1.3), we provide a lower bound on the minimum cost of P1 based on Algorithm 1, in which the ramping constraint is removed, i.e., $r=1$. As deriving an optimal control policy of $\mathbf{P 1}$ may require accurate system statistics or simply be intractable, we will use this lower bound as a benchmark for performance comparison in simulation. The performance gap between our algorithm and this lower bound serves as an upper bound on the performance gap between our algorithm and an optimal algorithm.

Note that, when the energy capacity $s_{i, \max }$ is predetermined, Theorem 1 still holds by setting $V \in\left(0, V_{\max }\right]$. From the definition of $V_{\max }$ given by (19) in Proposition 1, the performance of Algorithm 1 is limited by the smallest DS unit.

\section{Simulation Results}

In this section, we present a numerical evaluation of the proposed algorithm and compare it with two benchmarks.

\section{A. Simulation Setup}

For the microgrid considered in simulation, unless otherwise specified, the following parameters are set as default. The length of each time slot is $10 \mathrm{~min}$. The amounts of the base loads $l_{b, t}$ and the flexible loads $l_{f, t}$ are uniformly distributed on $[5,25] \mathrm{kWh}$, and the portion of the unsatisfied flexible loads $\alpha$ is 0.5 . The aggregator is connected with $N=30$ RGs. For each on-site DS unit, we set the maximum discharging and charging amounts as $\left|x_{i, \min }\right|=x_{i, \max }=1.1 \mathrm{kWh}$ by assuming the DS unit charging/discharging rate to be $6.6 \mathrm{~kW}$ (three-phase, level II) [15]. Since the model of the degradation cost function is proprietary and unavailable, in simulation we set $D_{i}(x)=10 x^{2}$ as an example. The renewable generation $a_{i, t}$ is uniformly distributed on $\left[0, a_{i, \max }\right]$ where $a_{i, \max }=1.1$ $\mathrm{kWh}$. For the $\mathrm{CG}$, we set the generation cost function as $C(x)=8 x$, the maximum output $g_{\max }=50 \mathrm{kWh}$, and the ramping coefficient $r=0.1$. The unit buying electricity price $p_{b, t}$ is uniformly distributed on $[10,12]$ cents $/ \mathrm{kWh}$, which is around the current mid-peak electricity price in Ontario [16]. The unit selling electricity price $p_{s, t}$ is uniformly distributed on $[4,6]$ cents/kWh, which is slightly below the current offpeak electricity price in Ontario [16]. The control parameter $V=1$ and the energy capacity $s_{i, \max }$ is given by (18).

\section{B. Comparison with Benchmarks}

For comparison, we provide two benchmarks. One is a greedy algorithm, which is myopic and aims to minimize the current system cost. The optimization problem of the greedy algorithm at time slot $t$ is formulated as follows.

$$
\begin{array}{cl}
\min _{\mathbf{u}_{t}} & w_{t} \\
\text { s.t. } & (3),(5)-(10),
\end{array}
$$




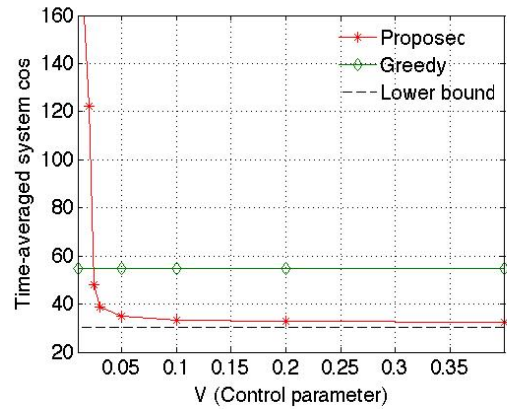

Fig. 2. Time-averaged system cost v.s. $V$.

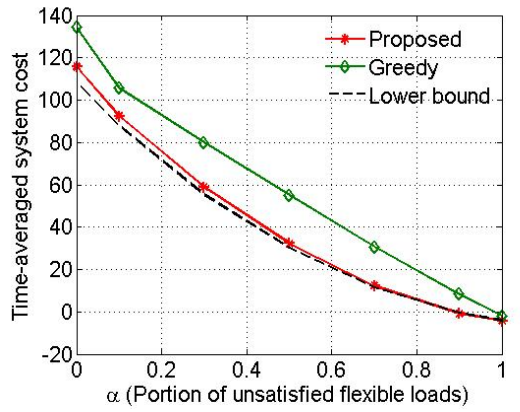

Fig. 3. Time-averaged system cost v.s. $\alpha$.

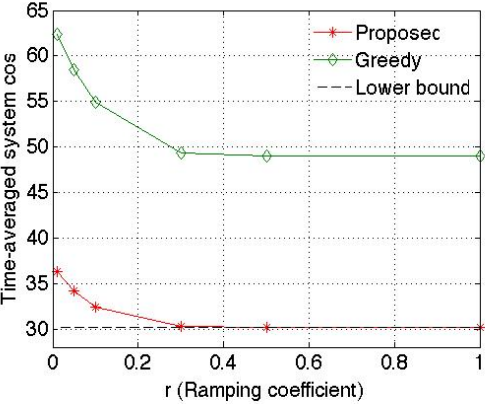

Fig. 4. Time-averaged system cost v.s. $r$.

$$
\begin{aligned}
& l_{b, t}+(1-\alpha) l_{f, t} \leq l_{m, t} \leq l_{b, t}+l_{f, t}, \\
& -s_{i, t} \leq x_{i, t} \leq s_{i, \max }-s_{i, t}
\end{aligned}
$$

We can show that the greedy algorithm does not employ the DS units because of its indifference to the future performance. For another benchmark, we consider the lower bound on the minimum cost in Theorem 1.3).

In Fig. 2, we depict the time-averaged system cost under various values of the control parameter $V$. For the proposed algorithm, the system cost drops quickly and then remains stable being very close to the lower bound. This observation reflects the efficiency of our algorithm and implies that using small DS units can be enough for being near optimum. In contrast, the performance of the greedy algorithm does not change with $V$. Moreover, the system cost under the greedy algorithm is about 1.7 times that under the proposed algorithm with $V \geq 0.1$.

In Fig. 3, we illustrate the effect of $\alpha$, the portion of the unsatisfied flexible loads, on the system cost. As expected, the system cost goes down as $\alpha$ rises. For the proposed algorithm, the marginal system cost decreases with $\alpha$, which indicates that the benefit of curtaining the flexible loads keeps on falling. Compared with the greedy algorithm, the proposed algorithm always achieves a lower system cost. Besides, it is close to the minimum cost for all cases.

In Fig. 4, we show the effect of the ramping constraint on the system cost. It can be seen that, the system cost is non-increasing with the ramping coefficient $r$. This is easy to understand since less expensive external energy resources are used with a looser ramping constraint. Furthermore, for both the proposed and the greedy algorithm, the marginal system cost goes down. In particular, the system cost cannot be decreased any further when $r \geq 0.3$. This indicates that the system supply is relatively sufficient in that region of $r$, and therefore it is unnecessary to further relax the ramping constraint. We also observe that, the proposed algorithm is superior to the greedy algorithm for all cases, and achieves the minimum cost when $r \geq 0.3$.

\section{CONCLUSION}

We have investigated the problem of real-time power balancing in a grid-connected microgrid, powered by one CG and multiple RGs each equipped with one DS unit. An aggregator operates the microgrid and aims to minimize the system cost by coordinating the supply side, demand side, and DS units. We have proposed a real-time algorithm that does not require any system statistics. Theoretically, we have characterized the gap between the system cost under our algorithm and the minimum cost, and shown the asymptotic optimality of our algorithm. In simulation, we have compared our algorithm with a greedy algorithm as well as a lower bound on the minimum cost and shown its efficiency.

\section{REFERENCES}

[1] N. Hatziargyriou, H. Asano, R. Iravani, and C. Marnay, "Microgrids," IEEE Power and Energ. Mag., vol. 5, no. 4, pp. 78-94, Jul.-Aug. 2007.

[2] A. Meier, Electric Power Systems: A Conceptual Introduction. WileyIEEE Press, 2006.

[3] L. Lu, J. Tu, C. Chau, M. Chen, and X. Lin, "Online energy generation scheduling for microgrids with intermittent energy sources and cogeneration," in Proc. ACM Sigmetrics, Jun. 2013.

[4] B. Narayanaswamy, V. Garg and T. Jayram, "Online optimization for the smart (micro) grid," in ACM e-Energy, May 2012.

[5] T. Chang, M. Alizadeh, and A. Scaglione, "Real-time power balancing via decentralized coordinated home energy scheduling," IEEE Trans. Smart Grid, vol. 4, no. 3, pp. 1490-1504, Sep. 2013.

[6] S. Sun, M. Dong, and B. Liang, "Real-time power balancing in electric grids with distributed storage," IEEE J. Sel. Topics Signal Process., IEEEXplore early access, Jun. 2014.

[7] _ - "Real-time welfare-maximizing regulation allocation in dynamic aggregator-EVs system," IEEE Trans. Smart Grid, vol. 5, no. 3, pp. 1397-1409, May 2014.

[8] Y. Zhang, N. Gatsis, and G. Giannakis, "Robust energy management for microgrids with high-penetration renewables," IEEE Trans. Sustainable Enery, vol. 4, no. 4, pp. 944-953, Oct. 2013.

[9] S. Salinas, M. Li, P. Li, and Y. Fu, "Dynamic energy management for the smart grid with distributed energy resources," IEEE Trans. Smart Grid, vol. 4, no. 4, pp. 2139-2150, Dec. 2013.

[10] P. Ramadass, B. Haran, R. White, and B. Popov, "Performance study of commercial $\mathrm{LiCoO} 2$ and spinel-based Li-ion cells," J. Power Sources, vol. 111, no. 2, pp. 210-220, Apr. 2002.

[11] M. Shahidehpour, H. Yamin, and Z. Li, Market Operations in Electric Power Systems: Forecasting, Scheduling, and Risk Management. WileyIEEE Press, 2002.

[12] M. Neely, Stochastic Network Optimization with Application to Communication and Queueing Systems. Morgan \& Claypool, 2010.

[13] L. Huang and M. Neely, "Utility optimal scheduling in energyharvesting networks," IEEE/ACM Trans. Netw., vol. 21, no. 4, pp. 11171130, Aug. 2013.

[14] S. Sun, M. Dong, and B. Liang, "Joint supply, demand, and energy storage management towards microgrid cost minimization," University of Toronto, Tech. Rep., 2014. [Online]. Available: http://www.comm.utoronto.ca/ ssun/mypapers/SGC14_tr.pdf

[15] A. Ipakchi and F. Albuyeh, "Grid of the future," IEEE Power Energy Mag., vol. 7, no. 2, pp. 52-62, 2009.

[16] "Electricity prices in ontario." [Online]. Available: http://www. ontarioenergyboard.ca/OEB/Consumers/Electricity/Electricity+Prices 